\title{
Performance Analysis of User-Centric Network Selection and Network-Centric Resource Allocation Approaches in Wireless Networks
}

\author{
Sahin Albayrak, Manzoor A. Khan^, Fikret Sivrikaya, \\ Ahmet C. Toker, and Cuong Troung \\ DAI-Labor, Technische Universität Berlin, Germany \\ ManzoorAhmed.Khan@dai-labor.de
}

\begin{abstract}
The possible leveraging of high deployment costs, and the possibility to increase revenue have also introduced the concept of network sharing between different operators. On the other hand, the realization of a user-centric paradigm in future heterogeneous wireless networks, which implies free and automatic choice among different available wireless and mobile access networks, will revolutionize future wireless networks. For this innovative concept to materialize a paradigm shift is required from a long-term contractual based service delivery to a shortterm contractual and dynamic service delivery. In this paper we formulate and compare the network-centric resource allocation and and user-centric network selection problems in a multi-operator scenario using two different flavors of game theory, namely bargaining games (for network-centric resource sharing) and multi-attribute auctions (for user-centric interface selection). We also compare the contributed solutions to one another to investigate their performance in terms of efficient resource allocation, call blocking probability, and user satisfaction 1
\end{abstract}

\section{Introduction}

We observe an increasingly heterogeneous landscape of wireless access technologies, including UMTS, GSM, WiFi, WiMAX, etc, which are specialized for different environments and user contexts. The development as well as the business cycles of these technologies can assure us that they will be available simultaneously for the years to come. Consequently, there has been significant research activity on the integration and inter-operability of these fundamentally different access technologies, which exhibit different service characteristics in terms of bandwidth, coverage, pricing, and Quality of Service (QoS) support. Common Radio Resource Management (CRRM) 1] is the concept that multiple such radio access technologies (RAT) can be combined in an operator network to diversify the service offer, as well as make use of trunking gains. The CRRM problem, which involves the allocation of call requests of different service types to the

\footnotetext{
* Corresponding author.

${ }^{1}$ This research activity is partially funded under the EU ICT project PERIMETER (FP7-224024).
} 
different Radio Access Networks (RANs), has been approached mostly from a single operator perspective, where different Radio Access Technologies (RATs) deployed as radio access networks belong to the same operator.

As in many areas of the networking field, application of game theory concepts to CRRM problem has been considered using both cooperative [2], and noncooperative / competitive 3] game models to obtain efficient resource allocation schemes. All these studies that apply game theory to CRRM are confined to a single network operator scenario. A parallel development in the communications industry is the emergence of the concept of network sharing [4], [5], where operators share RANs to leverage investment, and to improve utilization of their investments. In this network-centric view, the decisions of resource allocation are solely coordinated and taken by the operators, with the aim of resource optimization. With the emergence of multi-interface terminals that run increasingly diversified applications with different QoS requirements, the simple resource allocation and connectivity issue has started to shift into a more rewarding resource allocation problem. The main concept of user-centric view in network selection is to place end users, and the applications running on their terminals, at the center of decision making with the aim of improving the user experience for all different service types, while ensuring efficient resource utilization.

Keeping the above mentioned multi-dimensional issues in view, we propose both network-centric resource allocation and user-centric interface selection solutions in this paper and evaluate their performance. In the network-centric domain, we formulate the interaction in terms of two games, the intra-operator and the inter-operator games. In the former, the RANs belonging to an operator play a bargaining game to share the bandwidth of an incoming application bandwidth request. If an operator needs extra bandwidth to support the service request, it does so by playing the second game with other operators who have excess bandwidth and and are willing to share.

In the user-centric interface selection domain, where the decision of network selection resides at the mobile terminal, we model the interaction between mobile terminal and network operators using auctioning theory. Earlier research in the direction of user-centric networking concentrates on defining users' utility without taking into account the operators' benefits, hence omitting the motivation to realize the user-centric $\mathrm{ABC}$ vision. In this work, we are trying to address this by modeling the negotiation between users and operators using auctions, and to define the utilities of both operators and users.

\subsection{Model and Assumptions}

We consider a region $R$ covered by various RANs owned by different operators. An area may be covered by a single RAN, by multiple RANs belonging to a single operator, or by multiple RANs belonging to multiple operators. We assume there are $m$ different operators and $n$ different RATs which are combined into RANs by the network operators. A consequence of this hierarchy is the different definition of load and congestion for the RAN, cost incurred and area that is covered by different RANs. 
Before presenting our network-centric and user-centric models for network selection and resource allocation, we introduce or clarify a set of terms that will be utilized in the rest of the paper:

RAN Congestion - A RAN is said to be in congestion region if its available bandwidth falls below some pre-defined threshold value.

Aggregated Congestion - An operator network is said to be in the aggregated congestion region in an area $a$ if the aggregated available bandwidth of the RANs belonging to the operator in area $a$ falls below some threshold value. Operators' Incurred Costs on Network Technologies - Operators' cost on technology mainly depends on radio equipment, backhaul transmission equipment, license fees, site build-outs, and installation of equipments. Such deployment costs are constants and can be normalized to user pool. Whereas the service management, operation and maintenance (O\&M) costs can be used to categorize users.

\section{Network-Centric Cooperative Resource Allocation}

In this section we consider the traditional telecom model where users have contractual agreements with a home operator and generate application bandwidth requests of different QoS classes. Upon initial access selection, which is not a part of this work, a user connects to the home network using some RAN belonging to its home operator first, and generates bandwidth requests for applications of different service classes. The home operator first allocates this bandwidth request to different home RANs in the coverage area. We assume there is a functional CRRM entity that coordinates RANs of an operator in the area. If the operator is experiencing aggregated congestion in the area where the user is located, it will not allocate the bandwidth right away, but will request additional bandwidth from foreign operators which have RANs in the area, and are willing to share bandwidth. We assume that operators are in contractual agreements with each other to share resources, in terms of service level agreements (SLA). The interaction between operators is monitored by an SLA broker, which is an independent neutral entity. After the interaction, the requested bandwidth is distributed among those operators who are willing to share bandwidth and their RANs are present in the area. Each operator treats its share of the bandwidth as a new bandwidth request.

We use cooperative games to formulate the resource allocation management problem in multi-operator heterogeneous wireless networks at two levels. The first step in which the requested bandwidth is shared between the RANs of the same operator is called the intra-operator resource allocation, and the second allocation step is called the inter-operator resource distribution. At the intraoperator level operator's RANs in an area bargain over the requests coming from users that belong to the operator. Intra-operator game is played in order to find an optimal distribution of divisible application bandwidth among RANS, and is based on our previous work [6]. The request is allocated to different RANs, and the utility function of different RANs is set to be the amount of allocated bandwidth above a certain disagreement point. 
Utility Function of Network Technologies - Let $U_{w, o}$ represent the utility function of technology $w \in W$ owned by operator $o, B_{o}^{a}(q)$ the vector of bandwidth offer $b_{o, w}^{a}$, and $c_{w, o}$ the incurring cost (specifically $\mathrm{O} \& \mathrm{M}$ ). Then the utility function of $w$ is given by: $U_{w, o}\left(Q_{o}^{a}(q)\right)=x_{w, o}^{a}(q) \pi_{w, o}-c_{w, o} \quad \forall b_{o}^{a}(q) \in B_{o}^{a}(q)$, where $x_{w, o}^{a}(q)$ represents the allocated bandwidth and $\pi_{w, o}$ the price per unit bandwidth over the technology $w$ in area $a$. Since we concentrate on fixed rate pricing, the operator charges the same amount over all technologies owned by itself, irrespective of the cost incurred on technology. However this utility function strengthens the concept of priorities over network technologies set by the operator for different services, which is reflected in the offered bandwidth. The upper bound on the allocation bandwidth is defined by the feasible set [6].

Utility Function of Network Operators - Intuitively, the aggregated utility of all network technologies constitutes the operator utility:

$$
U_{o \in O}\left(Q_{o}^{a}(q)\right)=\sum_{w \in W} U_{w, o}\left(Q_{o}^{a}(q)\right) \quad \forall b_{o, w}^{a}(q) \in B_{o}^{a}(q) .
$$

If a bandwidth request cannot be fulfilled by the RANs of a home operator, then the inter-operator game is played. The inter-operator resource distribution problem is formulated such that each operator present in a coverage area bargains over the additional (excessive) bandwidth requests.

We model the allocation and distribution problems as bankruptcy problems and obtain the utility distribution rules. These rules dictate the allocation of requested bandwidth to RANs and the distribution of excess bandwidth to operators. We employ game theoretic approach of bargaining and the KSBS to obtain the allocation and distribution rules. The distribution rule is enforced by the SLA-broker, whereas the allocation rule is enforced by the CRRM manager. For computing the bandwidth offers in intra-operator games, we use the solution of proportional allocation rule to zero-associated bargaining problem presented in our previous paper 6].

For the inter-operator game, let $\bar{r}_{o}^{a}(q)$ represent the excess bandwidth request for a service class $q$ that an operator $o$ cannot fulfill, and would like to offer in the inter-operator game to other operators. Moreover, let the vector $\bar{Q}^{a}(q)$ represent all these requests from different operators in the region that are in aggregated congested regions. The operators play the game by making a bandwidth offer $b_{o}^{a}$, which can be grouped into the vector $B_{o}^{a}(q)$. The bargaining comes up with allocation of requested bandwidth to different operators, $x_{o}^{a}$, which should be a member of the compact and convex feasibility set:

$$
S\left(\bar{Q}^{a}(q), B_{o}^{a}(q)\right)=\left\{x_{o}^{a}: x_{o}^{a} \leq b_{o}^{a}, \sum_{i \in O} x_{i}^{a} \leq \sum_{i \in O} \bar{Q}^{a}(q)\right\}
$$

Let $C_{o}$ represent the aggregated capacity of operator $o$, which is calculated from the capacities of the RANs belonging to operator $o$ in that area. Similarly the condition for the bankruptcy formulation is: $\sum_{i \epsilon O} \bar{r}_{i}^{a}(q) \leq \sum_{i \epsilon O} b_{i}^{a} \leq$ $\sum_{i \epsilon O} C_{i}$. Contrary to the intra-operator game the disagreement point $D(q)=$ $\left(d_{1}(q), \ldots, d_{n}(q)\right) \in \mathbb{R}^{n}$ is calculated from the bandwidth requests and offers of 
the operators. To depict a realistic scenario we select the disagreement point as the characteristic function in our bankruptcy problem at inter-operator level. The characteristic function of a bargaining problem is defined as the amount of utility conceded to a player by all other players. operators. That is, for an operator $i$ and foreign operators $\forall j \neq i$ the disagreement bandwidth is given by $d_{i}(q)=\max \left\{0 ; \sum_{k \in O} \bar{r}_{k}^{a}(q)-\sum_{i \neq j} b_{j}^{a}(q)\right\}$.

Let $X^{a}=\left(x_{1}^{a}, \ldots, x_{m}^{a}\right)$ denote the solution obtained by applying KSBS to our inter-operator bargaining problem. Then $X^{a}=F^{K S}\left(S\left(\bar{Q}^{a}(q), B^{a}(q)\right), D(q)\right)$, which represents a $D$ associated bargaining problem. Recommendations made by $K S B S$ when applied to $D$ associated bargaining problem coincides by adjusted proportional distribution rule [7. This distribution rule is applied by the SLA broker.

$$
x_{o}^{a}=d_{o}(q)+\frac{\left(b_{o}^{a}(q)-d_{o}(q)\right)}{\sum_{i \in O}\left(b_{i}^{a}(q)-d_{i}(q)\right)} \cdot\left(\sum_{i \in O} \bar{r}_{i}^{a}(q)-\sum_{i \in O} d_{i}(q)\right) .
$$

The operator sums the most up-to-date bandwidth offers from the RANs in the area for the service class. Then this aggregated offer is scaled with the motivation factor of the operator $0 \leq \mu_{o} \leq 1$. By setting this factor, the operator is able to adjust the cooperative nature of its strategy. There is an incentive for cooperative behavior as operators can allocate unused bandwidth to increase utilization and therefore revenues. Then we have $b_{o}^{a}(q)=\mu_{o} \cdot \sum_{i \in W_{o}^{a}} b_{o, i}^{a}(q)$, where $b_{o, i}^{a}(q)$ represents bandwidth offer by network technology $w \in W_{o}^{a}$.

\section{User-Centric Multi-attribute Auction Based Interface Selection}

For a study of user-centric interface selection problem that is comparable to our network-centric resource allocation model, we consider user terminals that are equipped with at least as many interfaces as the network technologies available in any given coverage area. Upon receiving a request for an application class, network operators available within the coverage area present service offers that satisfy the minimum application requirements, which are evaluated by the users' expected values of different parameters. Parameters include technology-specific, application-specific and user-specific preferences. Users select the network technology / operator for any application whose offer matches closest to the user expectation.

In this section we formally define our user-centric network selection model as a multi-attribute auction game using the sealed bid second price Vickery auction format. For game-theory basics specific to this paper, our previous work [6], 8] can be referred. Let $U=\left\{u_{1}^{a}, \ldots, u_{n}^{a}\right\}$ be the set of users, $O_{w}^{a}=\left\{o_{1}^{a}, o_{2}^{a}, \ldots, o_{m}^{a}\right\}$ be the set of network operators, where $w$ is the network technology in area $a$ and $\left\{\left(r^{a}(q), \theta_{k}\right) \mid r^{a}(q) \in Q^{\prime}, \theta_{k} \in \theta\right\}$ be the user request within coverage area $a$, 
where $Q^{\prime}$ represent the set of application classes, which in turn is characterized by different amount of required bandwidth resource. $\theta$ defines the vector of various QoS attributes and expected ranges of these attributes for user request and $r^{a}(q)$ is the required bandwidth by application of QoS class $q$. In our model of user-centric interface selection, buyers are analogous to application users, sellers to network operators of available network technologies within coverage area $a$ and the requested bandwidth with associated attributes is considered as auctioned item. For any application request the user announces intention to acquire the item $\left(r^{a}(q), \theta_{k}\right)$ through the auctioneer residing on user terminal. Auctioneer broadcasts this request in the coverage area $a$, which is received by the operators owning network technologies in that area. Upon receiving this request the operator(s) submit their bids that identify the offer against the requested bandwidth.

The candidate operators create bids after they receive the broadcasted application bandwidth request by auctioneer in that area. Let the bid $b$ by operator technology $w$ be $b i_{w, o}^{a}$, which is given by the tuple:

$$
b i_{w, o}^{a}\left(r^{a}(q), \theta_{k}\right)=\left(\left(r^{a}(q), \theta_{k}\right), \pi_{w, o}^{a}\right)
$$

where $b i_{w, o}^{a}\left(r^{a}(q), \theta_{k}\right)$ is bid offer from operator $o$ through network technology $w$ in an area $a$ against the application required bandwidth $r^{a}(q)$, associated attribute vector $\theta_{k}$ and $\pi_{w, o}^{a}$ is per unit bandwidth payment. Both users and network operators are characterized by their utility functions. The bid formation is dictated by the operator utility function. Framing this simply, we can say that the bidding mechanism is influenced by candidate operators' strategies that increase their payoffs, before we define the utility function of a network operator. let us assume that $D=\max _{\{\bar{w}, \bar{o} \neq o\}} b i_{\bar{w}, \bar{o}}\left(\left(r^{a}(q), \theta_{k}\right), \pi_{\bar{w}, \bar{o}}\right)$ be the maximum suitable bid if operator $o \in O$ does not participate in the game. We also assume that the value of every QoS attribute is the simple linear function of cost incurred by network operator, meaning thereby cost increases linearly with increasing value of any QoS attribute in the direction of improving QoS. We also know that the operator receives amount against its extended service(bandwidth in our case) on per unit basis. Thus the utility function of operator in user-centric interface selection will be:

$$
U_{w, o}\left(r^{a}(q), \theta_{k}\right)=\left\{\begin{array}{l}
\left(\gamma_{q}, \theta_{k}\right) \pi_{w, o}^{a}-\sum_{k \in \theta} c_{w, o}\left(\theta_{k}\right) \text { if } D \leq b i_{w}\left(\theta_{k}\right) \\
0 \text { if } D \geq b i_{w}\left(\theta_{k}\right) \\
\lambda\left(\left(r^{a}(q), \theta_{k}\right) \pi_{w, o}^{a}-\sum_{k \in \theta} c_{w, o}\left(\theta_{k}\right)\right) \text { if } D=b i_{w}\left(\theta_{k}\right)
\end{array}\right.
$$

where $c_{w, o}\left(\theta_{k}\right)$ is the cost incurred on a single attribute value and we assume it to be the linear function of attribute values, meaning thereby increasing / decreasing the values of attribute that result in better user perceived QoS increases the network cost linearly. $\sum_{k \in \theta} c_{w, o}\left(\theta_{k}\right)$ is the operators' reservation price for service. $\lambda$ is tie breaking co-efficient that can take any value $\{0,1\}$ randomly.

It is straightforward to prove that the formulated auction is strategy proof, where each operator (bidder) maximizes its utility by bidding truthfully. The 
following lemmas follow from similar proof constructs given in [910], which we omit here due to space restriction.

Lemma-1 - For every bid bi $i_{w, o}^{a} \neq \widehat{b i_{w, o}^{a}}$ of an operator o $\in O$ there is a bidding profile $b i_{\{-w,-o\}}$ of other operators such that $u_{w, o}\left(b i_{\{-w,-o\}}^{a}, b i_{w, o}^{a}\right)<$ $u_{w, o}\left(b i_{\{-w,-o\}}^{a}, \widehat{b i_{w, o}^{a}}\right)$, where $b i_{w, o}^{a}$ is operator $o^{\prime} s$ bid with false valuation of request and $\widehat{b_{w, o}^{a}}$ represents the bid with true valuation.

Lemma-2 - In the strategy proof auction and for any value of offered bandwidth and associated $Q o S$ attributes $\left(r^{a}(q), \theta\right)=\operatorname{argmax}_{\left(r^{a}(q), \theta\right)}\left(\left(r^{a}(q), \theta\right) \pi_{w, o}^{a}-\right.$ $\left.\sum_{k \in \theta} c_{w, o}\left(\theta_{k}\right)\right)$ holds, where $\left(r^{a}(q), \theta\right) \pi_{w, o}^{a} \in \bigcup_{\theta, r^{a}(q) \in Q}$ which maximizes bidder's utility based on payment $\pi_{w, o}$.

Winning bid is the consequence of user satisfaction. The degree of users satisfaction is translated using user utility function, which captures users' preference relationship over various QoS attributes and their behavior towards the amount of offered bandwidth. Since QoS attribute vector is $n$-dimensional, therefore user preference to every single attribute is represented by assigning weights to attributes. User evaluate amount of offered bandwidth and each relevant attribute quoted in the offer from network provider using its utility function(scoring function) and compute the utility of overall bid by using weighted sum over each utilities attained for each individual attribute and the utility obtained from the offered bandwidth as:

$$
U_{i}\left(b i_{w, o}^{a}\left(r^{a}(q), \theta_{k}\right)\right)=\frac{u_{i}\left(r(q)^{o}\right)}{\sum_{k \in \theta} \psi_{k} \zeta_{w, o}\left(\theta_{k}\right)}-C_{i}\left(r^{a}(q), \theta_{k}\right)
$$

where $\zeta_{w, o}\left(\theta_{k}\right)$ represents the normalized value of each associated attribute $\theta_{k}$, $\zeta$ can be normalized in various expectancies i) the smaller the better, ii) the nominal the better, and iii) the greater the better. $\pi_{w}$ represents the payment per unit bandwidth over network $w, \psi_{k}$ is the user assigned weight to attribute $k$, and $u_{i}\left(r(q)^{o}\right)$ is the utility obtained by the offered bandwidth. The application utility is given by [1] 12

$$
u_{i}\left(\gamma_{q}^{o}\right)= \begin{cases}0 & \text { if } \gamma_{f} \leq \gamma_{\min } \\ u_{0}+\alpha \frac{1-e^{-\beta\left(\gamma_{q}^{o}-\gamma_{\min }\right)}}{1-e^{-\beta\left(\gamma_{q}-\gamma_{\min }\right)}} & \text { if } \gamma_{\min } \leq \gamma_{f} \leq \gamma_{\max } \\ u_{0}+\alpha & \text { if } \gamma_{f} \geq \gamma_{\max }\end{cases}
$$

where $\gamma_{\min }, \gamma_{\max }$ are minimum and maximum required application bandwidths respectively. $u_{0}$ is the private valuation of user, which in our case is the minimum application required bandwidth.i.e, for any value of offered bandwidth that is less than minimum application required bandwidth, users' utility is equal to zero. A careful look at the utility curves in [8] reveals that utility is concave for different services like video, audio and data. The assessment of different weights to various associated QoS attributes are of prime importance for decision of optimal interface selection. Various proposed techniques that compute these 
Table 1. QoS parameters and ranges

\begin{tabular}{llll}
\hline Parameters & Range & Score Category \\
\hline \multirow{2}{*}{ Delay } & $70 \mathrm{~ms} \sim 100 \mathrm{~ms}$ & 10 & Excellent \\
& $100 \mathrm{~ms} \sim 150 \mathrm{~ms} 8$ & Good \\
& $150 \mathrm{~ms} \sim 200 \mathrm{~ms} 6$ & Fair \\
\hline \multirow{2}{*}{ Jitter } & $30 \mathrm{~ms} \sim 50 \mathrm{~ms}$ & 10 & Excellent \\
& $50 \mathrm{~ms} \sim 60 \mathrm{~ms}$ & 5 & Good \\
& $60 \mathrm{~ms} \sim 70 \mathrm{~ms}$ & 3 & Fair \\
\hline \multirow{2}{*}{ Packet Loss } & $\sim 10^{-6} \sim 10^{-5}$ & 7 & Excellent \\
Rate & $10^{-5} \sim 10^{-4}$ & 4 & Fair \\
\hline
\end{tabular}

Table 2. Predefined offer bandwidth

\begin{tabular}{l||c|c|c}
\hline Class & GSM & WiMAX & UMTS \\
\hline \hline Voice & 500 & 240 & 400 \\
\hline Data & 900 & 1000 & 800 \\
\hline Video & 500 & 1600 & 500 \\
\hline
\end{tabular}

weights include Analytical Hierarchy Process (AHP), Fuzzy sets, Multi-attribute Utility Theory (MAUT), Smart Multi-attribute Rating Technique (SMART), etc. $C_{i}\left(r^{a}(q), \theta_{k}\right)$ in (5) represents the cost of service paid by user in terms of price. Auctioneer evaluates the offered service price as $C_{i}\left(\gamma_{q}, \theta_{k}\right)=\frac{\pi_{w, o}^{a}}{\pi_{i}^{\text {max }}}$, where the user specifies her maximum valuation of the service through $\pi_{i}^{\max }$.

For a typical user it is difficult to describe technically the preferences over communication parameters for any application. This can be addressed by providing a Graphical User Interface (GUI) to the users, as in [13, which takes the following inputs: i) service request class - Data, Video, Voice; ii) service preferred quality - Excellent, Good, Fair; iii) Service price preferences - Always Cheapest, Indifferent. Once these inputs are specified, they need to be translated into technical communication requirements. In this context, Table 1 [14 is used in order to map user defined service preferred quality to the technical communication parameters:

An auctioneer computes the wining bid from the matrix such that the wining bid maximizes the users' utility, i.e. by (5) . Auctioneer also determines the price that user pays to the winning network operator for the service. However the costs posed by user-centric network selection solution in terms of frequent handover is addressed by using fuzzy logic approach, explained in our earlier work [8].

\section{Results and Analysis}

In order to evaluate our approach for both network-centric resource allocation and user-centric interface selection, we have developed our own Java-based discrete event simulator, which generates user defined network operators, access technologies, users, different application quality classes and coverage areas with user defined RANs belonging to different operators [6]. We investigate the performance of our approach in randomly generated coverage areas for multi-operator heterogeneous wireless networks, consisting of GSM, UMTS, and WiMAX technologies.

We assume that a GSM RAN has a capacity of $4.500 \mathrm{kbps}$, UMTS 12.000 kbps, and WiMAX 20.000 kbps. The RAN overload thresholds are set to $10 \%$ for 


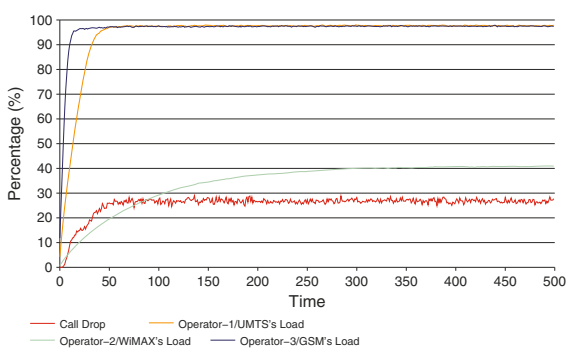

(a) Non-Cooperative Scenario

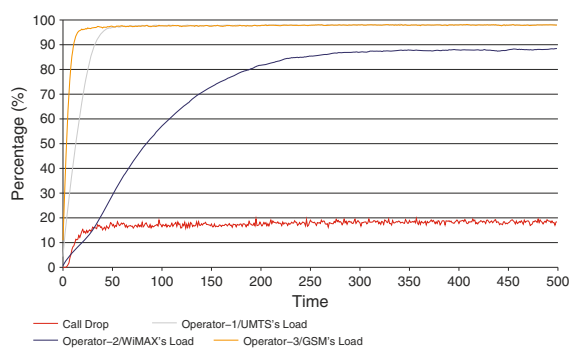

(b) Selective Cooperative Scenario

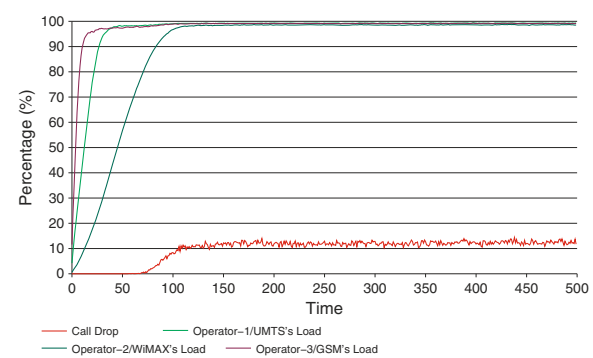

(c) Fully Cooperative Scenario

Fig. 1. Resource utilization and call blocking rate for different cooperation levels

UMTS and GSM, and to 3\% for WiMAX. Users request generation is modeled by a poisson process, call holding time is exponentially distributed, and the service class is chosen randomly among voice, data, and video uniformly. The sizes of the requests are assumed to be static, and are $60 \mathrm{kbps}, 150 \mathrm{kbps}$, and 500 kbps for voice, data, and video respectively. After the allocation and distribution algorithms, the allocated bandwidths are subtracted from the bandwidth pools of the RANs. The operators share the same predefined offered bandwidth values in kbps, which are given in Table 2. We assume a coverage area $a$ covered by three technologies owned by three different operators, such that operator-1 has UMTS, operator-2 has WiMAX and operator-3 has GSM in the area.

To investigate the game-theoretic network-centric resource allocation, we assume that there are different number of users who have contractual agreements with different available operators. Users of each operator are assumed to generate service requests according to different poisson distributions with means $\tilde{\mu}_{o p-1}$, $\tilde{\mu}_{o p-2}$ and $\tilde{\mu}_{o p-3}$. For the network-centric allocation case, we simulate operators' strategies when they cooperate under under-utilized and in-congestion conditions, or when they are non-cooperative. We assume that the requests are generated using $\tilde{\mu}_{o p-1}=2, \tilde{\mu}_{o p-2}=1$, and $\tilde{\mu}_{o p-1}=2$. Here we discern three cases based on different motivation factors of the involved operators. In the cooperative case all operators have a motivation factor of 1 . In the selective cooperative case operators 1 and 2 have a motivation factor of 1 , and operator- 3 has a motivation factor of 0 . In the non-cooperative case all operators have a motivation factor of 0 . 
Fig. 1(a) represents the non-cooperative case, which depicts that operator-2 is under-utilized and the aggregated call blocking is around $30 \%$ owing to the fact that all the operators are non-cooperative. In Fig. 1(b) the selective cooperative case, call blocking is reduced by $30 \%$ and resource utilization of operator- 2 is increased by almost $100 \%$ when compared with the non-cooperative case. In the fully cooperative case, as shown in Fig. 1(c), the aggregated call blocking percentage is reduced to almost $10 \%$ whereas the resources of all operators in the area are fully utilized. This demonstrates the performance of proposed cooperative approach in terms of resource utilization, call blocking and ultimately the revenue of operators (which is a function of utilization).

In order to investigate the performance of user-centric interface selection gain of users in terms of their utility, we consider users randomly generate requests of different quality of service class audio, video and data specifying service preferred QoS i.e., excellent, good and fair. These requests are generated using poisson distribution with the mean $\tilde{\mu}_{u c}$. To make this scenario comparable to network-centric scenario, the mean $\tilde{\mu}_{u c}$ is kept as $\tilde{\mu}_{u c}=\sum_{i=1}^{3} \tilde{\mu}_{o p-i}$, meaning thereby the same number of users' requests are generated in the coverage area as in network-centric case, but now users have no long-term contractual agreements with operator, and they are free to get associated with any operator. Operators' offer vary over time specially in terms of offered prices and offered QoS attribute values owing to the network condition, congestion and operator preferences. This results in triggering the interface selection decision. Performance of our auction-based interface selection approach is evaluated against the networkcentric approach in terms of average values over user's throughput, delay, jitter, packet-loss and payed price over 500 simulation instances are depicted in Fig. $2(\mathrm{a})$ As can be seen that auction-based approach performs better by about $10 \%$ in user throughput and about $9 \%$ in the price.

The improvement is achieved at the cost of frequent handovers, which is revealed in the CDF curve in Fig. 2(c). To address the tradeoff between handover frequency and $\mathrm{ABC}$ goal, we also simulate the auction-based with fuzzy approach [8]. The results reveal that in terms of both throughput and price, auction-based with fuzzy approach performs almost the same as auction-based approach, but with almost half time the handovers as shown in Fig. 2(c), In parallel a comparison over the price is shown in the CDF graph in Fig. 2(b).

We also compare auction based interface selection in terms of call blocking rate and resource utilization with our game-theoretic network-centric resource allocation. Fig. 2(d) reveals that user-centric approach performs better in terms of resource utilization, owing to the fact that users are always able to connect to any operator if blocked by one operator. Comparing the results in Fig. 2(d) with our game-theoretic approach in Fig. 1(c) it can be concluded that user-centric approach behaves somewhat similar to the cooperative game theoretic approach in terms of resource utilization and call blocking, and performs better than other network-centric approaches (please refer to [6] for the related comparison). This result dictates that auction-based user-centric and cooperative network-centric 


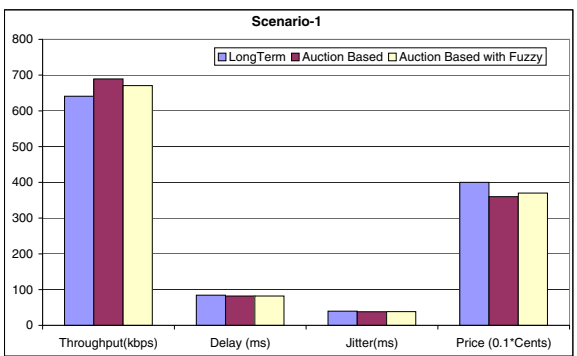

(a) Throughput, QoS and price comparisons of long-term-based approach against auction-based and auction-based with fuzzy approaches

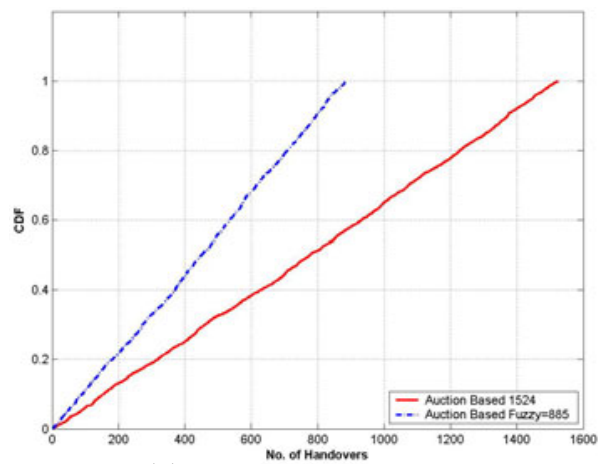

(c) $\mathrm{CDF}$ curve of price

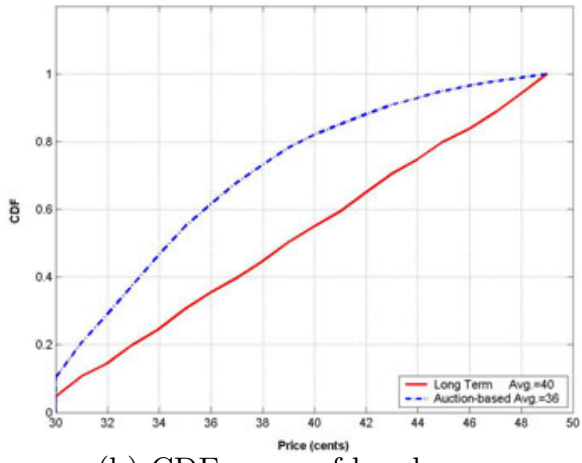

(b) CDF curve of handovers

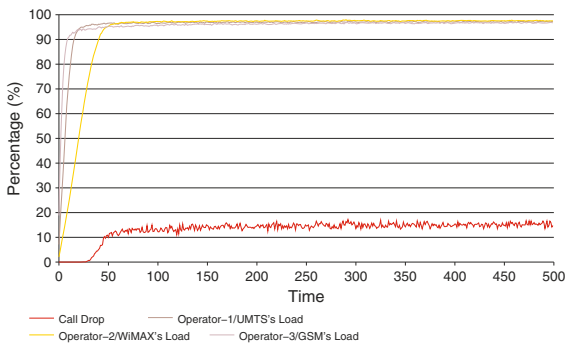

(d) User-centric Interface Selection

Fig. 2. Results of User-Centric Interface Selection

approaches provide similar incentives in terms of better resource utilization, less call blocking, and more satisfied users. Moreover, assuming that efficient resource utilization ultimately means higher revenues for the operators, these two approaches also increase operator satisfaction.

\section{Conclusion}

In this paper we have presented game theoretic formulations of network-centric multi-operator CRRM and user-centric network selection problems. On the one hand, at the network level CRRM manager employs an allocation rule to distribute bandwidth requests from user applications among operator RANs based on the offers they make. On the other hand, we present the network selection decision mechanism in a user-centric network scenario where users dynamically select the best available network. We have provided comparisons for both approaches with one another in terms of the gain of both stake-holders, 
i.e. satisfaction and reduced costs for users and resource utilization and call blocking probability for operators. The results signal that user-centric (auctionbased) network selection has a significant potential for satisfying both user and operators, thereby improving user experience and operators' resource utilization. Interestingly, the results for auction-based network selection in the usercentric scenario resembles to those of the cooperative game-theoretic approach in network-centric scenario. One can intuitively infer that these two approaches also result in higher revenues for operators compared to other approaches, although the actual revenue is dependent on the pricing scheme adopted by the operators. As a future work, we plan to incorporate the operators' pricing schemes into our model and investigate the dependencies between pricing, resource utilization, operator revenue, and user satisfaction.

\section{References}

1. Perez-Romero, J., Sallent, O., Agusti, R., Karlsson, P., Barbaresi, A., Wang, L., Casadevall, F., Dohler, M., Gonzalez, H., Cabral-Pinto, F.: Common radio resource management: functional models and implementation requirements. In: IEEE 16th International Symposium on Personal, Indoor and Mobile Radio Communications, PIMRC 2005, vol. 3, pp. 2067-2071 (2005)

2. Niyato, D., Hossain, E.: A cooperative game framework for bandwidth allocation in $4 \mathrm{~g}$ heterogeneous wireless networks. In: Proc. IEEE International Conf. on Communications ICC '06, vol. 9, pp. 4357-4362 (2006)

3. Das, S., Lin, H., Chatterjee, M.: An econometric model for resource management in competitive wireless data networks. IEEE Network 18(6), 20-26 (2004)

4. Beckman, C., Smith, G.: Shared networks: making wireless communication affordable. IEEE Wireless Communications, [see also IEEE Personal Communications] 12(2), 78-85 (2005)

5. Hultell, J., Johansson, K., Markendahl, J.: Business models and resource management for shared wireless networks. In: 2004 IEEE 60th Vehicular Technology Conference, VTC 2004-Fall, vol. 5, pp. 3393-3397 (2004)

6. Khan, M.A., Trong, C., Geithner, T., Sivrikaya, F., Albayrak, S.: Network level cooperation for resource allocation in future wireless networks. In: Proceedings of the IFIP Wireless Days Conference '08 (2008)

7. Dagan, N., Volij, O.: The bankruptcy problem: a cooperative bargaining approach. Nir Dagan, Economic theory and game theory (1993)

8. Khan, M.A., Sivrikaya, F., Mengal, K., Albayrak, S.: Auction based resource allocation in heterogeneous wireless networks. In: Proceedings of the IFIP Wireless Days Conference '09 (2009)

9. Nisan, N., Roughgarden, T., Tardos, E., Vazirani, V.V. (eds.): Algorithmic Game Theory, September 2007. Cambridge University Press, Cambridge (2007)

10. Suyama, T., Yokoo, M.: Strategy/false-name proof protocols for combinatorial multi-attribute procurement auction. In: AAMAS '04: Proceedings of the Third International Joint Conference on Autonomous Agents and Multiagent Systems, pp. 160-167. IEEE Computer Society, Washington (2004)

11. Wu, Z., Yin, Q.: A heuristic for bandwidth allocation and management to maximize user satisfaction degree on multiple mpls paths. In: 3rd IEEE Consumer Communications and Networking Conference, CCNC 2006, January 2006, vol. 1, pp. 35-39 (2006) 
12. Chan, H., Fan, P., Cao, Z.: A utility-based network selection scheme for multiple services in heterogeneous networks. In: 2005 International Conference on Wireless Networks, Communications and Mobile Computing, June 2005, vol. 2, pp. 1175 $1180(2005)$

13. Toker, A.C., Cleary, F., Fiedler, M., Ridel, L., Yavuz, B.: Perimeter: Privacypreserving contract-less, user centric, seamless roaming for always best connected future internet. In: 22th World Wireless Research Forum (2009)

14. Kim, H.J., Lee, D.H., Lee, J.M., Lee, K.H., Lyu, W., Choi, S.G.: The qoe evaluation method through the qos-qoe correlation model. In: Fourth International Conference on Networked Computing and Advanced Information Management, NCM '08, September 2008, vol. 2, pp. 719-725 (2008) 